\title{
Effects of phonon interference through long range interatomic bonds on thermal interface conductance
}

\author{
Haoxue Han $^{1,2}$, Lei Feng ${ }^{3}$, Shiyun Xiong ${ }^{2}$, Takuma Shiga ${ }^{3}$, Junichiro Shiomi ${ }^{3}$, \\ Sebastian Volz ${ }^{2}$, and Yuriy A. Kosevich ${ }^{4}$ \\ ${ }^{I}$ Theoretische Physikalische Chemie, Eduard-Zintl-Institut für Anorganische und Physikalische Chemie, Technische \\ Universität Darmstadt. 4 Alarich-Weiss-Straße, Darmstadt 64287, Germany \\ E-mail: haoxue.han@ecp.fr \\ ${ }^{2}$ Laboratoire EM2C, CNRS, CentraleSupélec, Université Paris-Saclay, Grande Voie des Vignes \\ 92295 Châtenay-Malabry cedex, France \\ ${ }^{3}$ Department of Mechanical Engineering, The University of Tokyo, 7-3-1 Hongo, Bunkyo, Tokyo, Japan \\ ${ }^{4}$ Semenov Institute of Chemical Physics, Russian Academy of Sciences, 4 Kosygin Str., Moscow 119991, Russia \\ E-mail: yukosevich@gmail.com
}

Received April 12, 2016, published online June 24, 2016

\begin{abstract}
We investigate the role of two-path destructive phonon interference induced by interatomic bonds beyond the nearest neighbor in the thermal conductance of a silicon-germanium-like metasurface. Controlled by the ratio between the second and first nearest-neighbor harmonic force constants, the thermal conductance across a germanium atomic plane in the silicon lattice exhibits a trend switch induced by the destructive interference of the nearest-neighbor phonon path with a direct path bypassing the defect atoms. We show that bypassing of the heavy isotope impurity is crucial to the realization of the local minimum in the thermal conductance. We highlight the effect of the second phonon path on the distinct behaviors of the dependence of the thermal conductance on the impurity mass ratio. All our conclusions are confirmed both by Green's Function calculations for the equivalent quasi-1D lattice models and by molecular dynamics simulations.
\end{abstract}

PACS: 65.80.-g Thermal properties of small particles, nanocrystals, nanotubes, and other related systems;

43.40.+s Structural acoustics and vibration;

63.20.-e Phonons in crystal lattices;

66.70.Lm Other systems such as ionic crystals, molecular crystals, nanotubes, etc.

Keywords: phonon interference, thermal conductance, metasurface, molecular dynamics.

\section{Introduction}

Kapitza measured the thermal boundary resistance at the interface of superfluid helium and a solid [1]. Kapitza resistance $R$, more generally referred to as thermal interface resistance, is defined as the ratio between the temperature difference $\Delta T$ at the interface over the heat flux per unit area $Q$ flowing across the interface,

$$
R=\frac{\Delta T}{Q} .
$$

To explain such thermal resistance at the boundary to helium, Khalatnikov proposed a model relating the transmission probability of phonon waves to their acoustic impedance of each medium, which is now known as the acoustic mismatch model (AMM) [2]. AMM predicts well the experimental measurements of the thermal boundary resistances for materials with relative small acoustic mismatch. However, the Khalatnikov formula overpredicts the Kapitza resistance at the solid-helium interface by two orders of magnitude. To alleviate this discrepancy between AMM and experiments, a diffuse mismatch model (DMM) was proposed to consider the density of vibrational (phonon) states in the calculation of transmission probability, since the model assumes a complete scattering of the incoming phonon waves [3]. Such scattering opens additional phonon channels for heat transfer and hence reduces the Kapitza resistance. 
Due to the wave nature of phonons, interference could play an important role in the thermal transport and hence impact the thermal interface resistance [4-9]. Destructive interference effects with total phonon reflection as a signature were discovered in acoustic systems $[10,11]$. Such an enhanced acoustic reflection was first described theoretically in Refs. 10 and 11 independently. Fellay et al. [10] studied the asymmetric profile in the phonon transmission through one-dimensional chains, in analogy with electron scattering. Reference 11 interpreted the reflection of an acoustic wave by using two-dimensional crystal defects for the destructive interference between two phonon paths. Phonon interference effects can be employed in manipulating the thermal transport in nanomaterials, hence understanding of phonon wave dynamics is helpful in improving the thermoelectric efficiency. The figure of merit $z T$ for thermoelectric conversion efficiency can be expressed as $z T=\sigma S^{2} T / \kappa$, where $S, T, \sigma$, and $\kappa$ are the Seebeck coefficient, temperature, electrical, and thermal conductivities, respectively. Thus, a low thermal conductivity is favorable for good thermoelectric performance. Recent efforts concentrated on reducing the thermal conductivity $\kappa$ via nanostructured materials with grain boundaries [12-14] and embedded nanoparticles [15-17]. Reducing $\kappa$ is often achieved by enhancing phonon scattering rate and thus diminishing the mean free path (MFP), which belongs to a particle description. Nevertheless, the role of destructive phonon-wave interferences remains to be well understood in the tailoring of thermal transport in a wave picture.

In the paper we investigate the role of the two-path destructive phonon interference induced by interatomic forces beyond the nearest neighbor on the thermal conductance of a silicon-germanium-like metasurface and a quasi-1D harmonic chain model. Controlled by the ratio between the second and first nearest-neighbor harmonic force constants, the thermal conductance across a germanium atomic plane in the silicon lattice exhibits a trend switch induced by the destructive interference of the nearest-neighbor phonon path with a direct path bypassing the defect atoms. We show that the heavy isotope impurity is crucial in the realization of the local minimum in the thermal conductance. We highlight the effect of the second phonon path on the distinct behaviors of the dependence of the thermal conductance on the impurity mass ratio.

\section{Phonon interference in thermal conductance}

The atomistic scheme of two-path phonon interference is illustrated in the insets of Fig. 1. In most of the lattices where the atoms are mainly coupled to each other through the nearest-neighbor bonds, the propagation path of phonon is restricted to the host-guest atom bonds when encountering an impurity atom. An additional phonon path becomes available when considering the second-nearestneighbor bonds between the host atoms on the two sides of
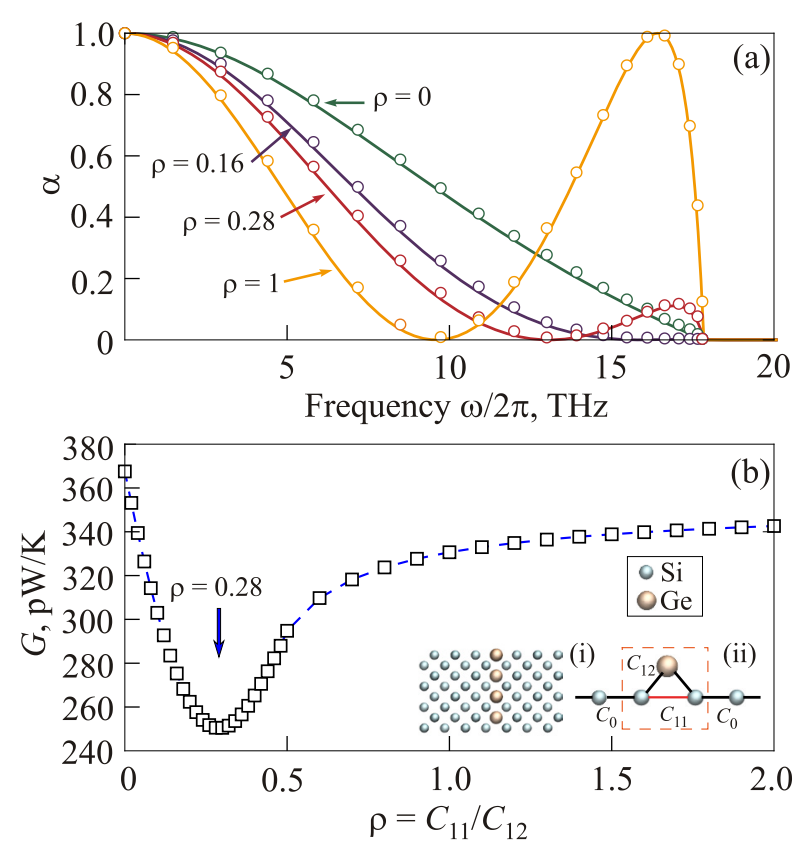

Fig. 1. (Color online) (a) Spectral transmission coefficients $\alpha(\omega, \rho)$ predicted by MD simulations (open circles) for a Si host crystal with a single atomic layer of Ge atoms and by Green's Function calculations for an equivalent quasi-1D model (solid lines). Only the longitudinal polarization is shown for the MD prediction. (b) Thermal conductance $G(\rho)$ versus the relative strength of the second nearest-neighbor bond $\rho$ at $T=300 \mathrm{~K}$. Open squares linked by a dashed line represent the thermal conductances corresponding to the bond ratios $\rho$. Inset ( $i$ ): host silicon lattice with a single $\langle 001\rangle$ atomic layer of guest $\mathrm{Ge}$ atoms. Inset ( ii ): quasi-1D tight-binding model which incorporates the second nearest-neighbor bonds $C_{11}$ bypassing the nearestneighbor bonds $C_{12}$ between the host atom with mass $m_{1}$ and the guest atom with mass $m_{2}$. The host atoms are coupled through the nearest-neighbor bonds $C_{0}$. Black (red) sticks represent the $\mathrm{NN}$ (second $\mathrm{NN}$ ) bonds. The region inside the red dashed rectangle is the scattering region.

the impurity atom in addition to the first path through the nearest-neighbor bonds linking the host and adjacent impurity atoms, as shown in Fig. 1. Destructive interferences will emerge due to the opening of the second phonon path that couples directly crystal layers adjacent to the defect atoms. We first investigate the intriguing role of such phonon interferences in the interfacial thermal conductance by using molecular dynamics (MD) modeling of the transmission of phonon wave-packets (WP) propagating in a silicon (Si) host lattice through a defect atomic plane of germanium (Ge), as shown in inset (i) of Fig. 1(b). Such WP modeling provides the per-phonon-mode energy transmission coefficient [9] (see Appendix 4). The spatial width $l$ (coherence length) of the WP is taken much larger than the wavelength $\lambda_{c}$ of the WP central frequency, corresponding to the plane-wave approximation $[7,8]$. The MD simulations were performed with the LAMMPS code package 
[18]. The covalent $\mathrm{Si}: \mathrm{Si} / \mathrm{Ge}: \mathrm{Ge} / \mathrm{Si}: \mathrm{Ge}$ interactions are modeled by the Stillinger-Weber (SW) potential [19].

The spectral transmission coefficients $\alpha(\omega, \rho)$ retrieved from MD simulations of a $\mathrm{Si}$ host crystal with a single atomic layer of Ge atoms are illustrated in Fig. 1(a), where the mass ratio of $\mathrm{Ge}$ and $\mathrm{Si}$ atoms $m_{\mathrm{Ge}} / m_{\mathrm{Si}}=2.6$ was used. $\rho$ is defined as the ratio of the second nearest-neighbor (NN) force constant $C_{11}$ of $\mathrm{Si}$ atoms and the first-NN force constant $C_{12}$ between $\mathrm{Si}$ and Ge atoms, $\rho=C_{11} / C_{12}$. $\rho$ measures the relative strength of the second $\mathrm{NN}$ interaction versus the NN force. Our First Principle calculations show that $\rho \approx 0.057$ in natural $\mathrm{SiGe}$ alloys where the nearest neighbor (NN) bond is $C_{12}=3.21 \mathrm{eV} / \AA^{2}\left(3.51 \mathrm{eV} / \AA^{2}\right)$ for the $\mathrm{Si}-\mathrm{Si}(\mathrm{Si}-\mathrm{Ge})$ atom pair, and the second-NN bond strength is $C_{11}=0.187 \mathrm{eV} / \AA^{2}\left(0.192 \mathrm{eV} / \AA^{2}\right)$ for the $\mathrm{Si}-\mathrm{Si}$ (Ge-Ge) pair (see Appendix 5). Empirical SW potential reproduces relatively precise NN bonds but its second $\mathrm{NN}$ bonds are negligible. When a single phonon path is available for phonons crossing the $\mathrm{Ge}$ atomic plane, i.e., $\rho=0$, the transmission coefficient $\alpha(\omega, \rho=0)$ monotonously decays as frequency increases with $\alpha(\omega=0)=1$ and $\alpha\left(\omega=\omega_{\max }\right)=0$. Relatively weak second NN interaction $\rho=0.16$ reduces the transmission on the whole spectrum but the effect is especially strong at short wavelengths when $\alpha(\omega \geq 15 \mathrm{THz}) \approx 0$. Such a remarkable drop in the transmission coefficient is due to the destructive interference between the two phonon paths: through the nearestneighbor $\mathrm{Si}-\mathrm{Ge}$ bonds and through the non-nearest-neighbor $\mathrm{Si}-\mathrm{Si}$ bonds which couple directly atomic layers adjacent to the defect plane [7]. For a larger $\rho=0.28$, a total transmission antiresonance emerges $\alpha(\omega=13 \mathrm{THz})=0$ followed by a local transmission maximum. When $\rho$ further strengthens, the total reflection shifts to longer wavelengths and the local maximum finally becomes a total transmission.

Figure 1(b) shows the thermal conductance $G$ vs $\rho$ at $T=300 \mathrm{~K} . G(\rho)$ is calculated by following the Landauerlike formalism [2]:

$$
G(\rho)=\int \sum_{v} \hbar \omega_{\mathbf{q}} \mathbf{v}_{\mathbf{q}}^{g, \perp} \alpha(\omega, \rho) \frac{\partial}{\partial T} n_{B E}(\mathbf{q}, T) \frac{d \mathbf{k}}{(2 \pi)^{3}},
$$

where $\mathbf{q}$ denotes the phonon mode $(\mathbf{k}, v), \mathbf{k}$ is the wave vector and $v$ is the phonon polarization. $v_{q}^{g, \perp}$ is the group velocity component perpendicular to the Ge atomic plane. $n_{B E}=\left[\exp \left(\hbar \omega_{\mathbf{q}} / k_{B} T\right)-1\right]^{-1}$ is the Bose-Einstein distribution of phonons, where $T$ refers to the mean temperature of the system, $k_{B}$ and $\hbar$ represent the Boltzmann and the reduced Planck's constants, respectively. The thermal conductance $G$ first decreases as the second phonon path strengthens, since the opening of the second phonon path through the second $\mathrm{NN}$ bonds $C_{11}$ interferes destructively with the first path through the NN bonds $C_{12}$, which induces a maximum reduction in $G$ of $33 \%$ at $\rho=0.28$. $G$ then starts to increase as the second NN bond further strength- ens, due to the raised phonon transmission at high frequencies, c.f. Fig. 1(a). The destructive interference is compensated by the very strong second phonon path at large $\rho$. To further understand the phonon antiresonances caused by the destructive interference between two phonon channels, we use an equivalent tight-binding model of a monatomic quasi-1D lattice of coupled harmonic oscillators, depicted in the inset (ii) of Fig. 1(b). The interatomic bonds could be adjusted to the same as in the $\mathrm{Si}-\mathrm{Ge}$ system to allow for a direct comparison. The phonon transmission is calculated by atomistic Green's Function (AGF) and the thermal conductance can be obtained by following the LandauerBüttiker formula:

$$
G=\int_{0}^{\omega_{\max }} \Xi(\omega) \frac{\partial}{\partial T} n_{B E} \hbar \omega \frac{d \omega}{2 \pi},
$$

where $\omega$ and $\omega_{\max }$ are the energy and the Debye frequencies. The transmission $\Xi(\omega)$ is obtained from a nonequilibrium Green's function approach [20] (see Appendix 6 for the derivation of the dynamical matrices). As shown in Fig. 1(a), the AGF prediction of the phonon transmission from the quasi-1D model agrees relatively well with that of the longitudinal phonons transmission from MD simulations in a $\mathrm{Si}-\mathrm{Ge}$ lattice.

We turn to the study of the phonon transport in the quasi-1D chain model in Fig. 1(b) with the second phonon path but without mass defect. The mass of the guest atom is the same as that of the host atoms, $m_{2}=m_{1}=m_{\mathrm{Si}}$. The phonon transmission through such a lattice is shown in Fig. 2(a) and the associated thermal conductance at $T=$ $=300 \mathrm{~K}$ is shown in Fig. 2(b). When there is only a single phonon path, i.e., $\rho=C_{11} / C_{12}=0$, the total transmission is recovered in the whole spectrum, $\alpha\left(\omega \in\left[0, \omega_{\max }\right)\right)=1$. When a weak second $\mathrm{NN}$ bond is available getting around the guest atom, a local minimum arises in the spectrum of high frequency phonons, whereas the transmission remains perfect both at the long-wave limit and close to the maximum frequency, while we always have $\alpha\left(\omega=\omega_{\max }\right)=0$. As $\rho$ increases, such local minimum shifts to higher frequencies and finally turns to a transmission antiresonance (complete reflection) at $\omega_{\max }$ for $\rho=0.5$. As $\rho$ increases, the total reflection remains and shifts to lower frequencies, as is found in Fig. 1(a). The thermal conductance in Fig. 4(b) shows a monotonous reduction as the second NN bond strengthens. The minimum in the thermal conductance, which was found in Fig. 1(b), has disappeared. Therefore, the presence of the heavy mass defect is crucial to realize such a minimal thermal conductance in the model incorporating the second phonon path, cf. Figs. 2(b) and 2(b), in contrast to the realization of the transmission antiresonance, which occurs in 2D metasurfaces with both light and heavy impurities, cf. Figs. 1(a) and 2(a). 

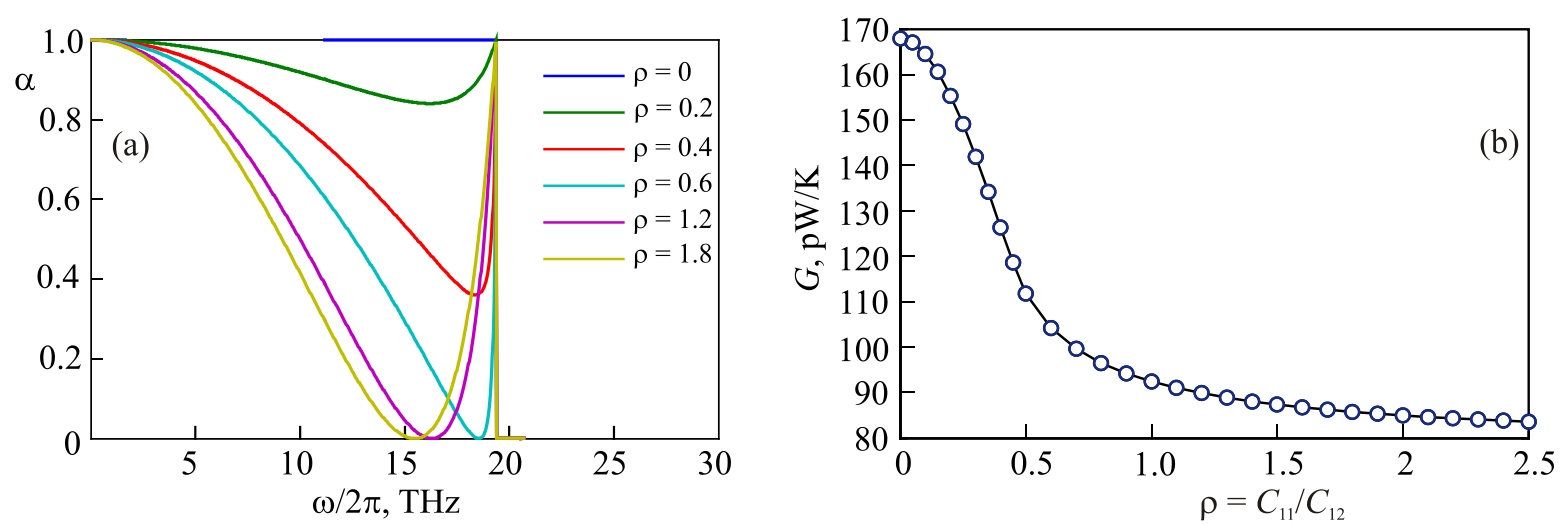

Fig. 2. (a) (Color online) Phonon transmission $\alpha$ of the quasi-1D model with the impurity mass $m_{2}=m_{1}=m_{\mathrm{Si}}$ in Fig. 1 vs frequency for different relative second NN bond strength $\rho$. (b) Thermal conductance $G$ vs the relative strength of the second NN bond $\rho$ at $T=300 \mathrm{~K}$.

So far, we have investigated the strength variation of second phonon path on the phonon transmission and thermal conductance. In a real experiment, it can be tricky to tune such second nearest neighbor forces. Nevertheless, it is rather easy to change the mass of the impurity atoms by choosing different isotopes of the host atoms. Moreover, we have shown that the minimum of thermal conductance can only be realized with heavy impurities. Hence it is informative to discuss the dependence on impurity mass of the phonon transport properties of a lattice incorporating additional phonon paths. To this purpose, we investigate the phonon transport in the quasi-1D chain model shown in Fig. 1(b) with the second phonon path by highlighting the role of the mass defect. We first study the case of a lattice with an isotope with the relative strength of the second NN bond $\rho=0.058$ which corresponds to the force constant obtained from our first principle calculations. In Fig. 3(a), we plot the phonon transmission in the quasi-1D model with an impurity atom for different impurity mass ratio $\rho_{m} \in[0.1,3]$, and the related thermal conductance versus $\rho_{m}$ in Fig. 3(b). It is clear that on the whole spectrum the phonon transmission first increases with $\rho_{m}$ in the range $\rho_{m} \in[0.1,0.93]$ and then decreases as the impurity mass further increases. The thermal conductance follows the same trend and reaches a maximum at $\rho_{m} \approx 0.93$. Such a behavior is rather straight ward to understand since for a weak second NN bond, the phonon transmission is recovered the best when the isotopic scattering is the least. A perfect transmission would be achieved for $\rho_{m}=1$ when only NN interaction is possible.

When the second $\mathrm{NN}$ bond $C_{11}$ is comparably strong with the $\mathrm{NN}$ bond $C_{12}=C_{0}$, the thermal conductance versus the impurity mass ratio $\rho_{m}$ cannot be continued from the limit of $C_{11} \approx 0$. In Figs. 4(a) and 4(b), we compare the phonon transmission and thermal conductance for impurity mass ratios $\rho_{m}=0.9,1.2$ and for the second $\mathrm{NN}$ bond strengths of $\rho=0.5,2$. The dependence of the thermal conductance on the impurity mass ratio can exhibit distinct behavior for different second $\mathrm{NN}$ bonds, as can be seen from Fig. 4(b). As the relative second $\mathrm{NN}$ bond strength increases from $\rho=0.5$ to $\rho=2$, the impurity-mass dependent thermal conductance $G\left(\rho_{m}\right)$ changes from a monotonous reduction to a more complicated curve with a local maximum at $\rho_{m}=0.9$ and a minimum at $\rho_{m}=1.2$.
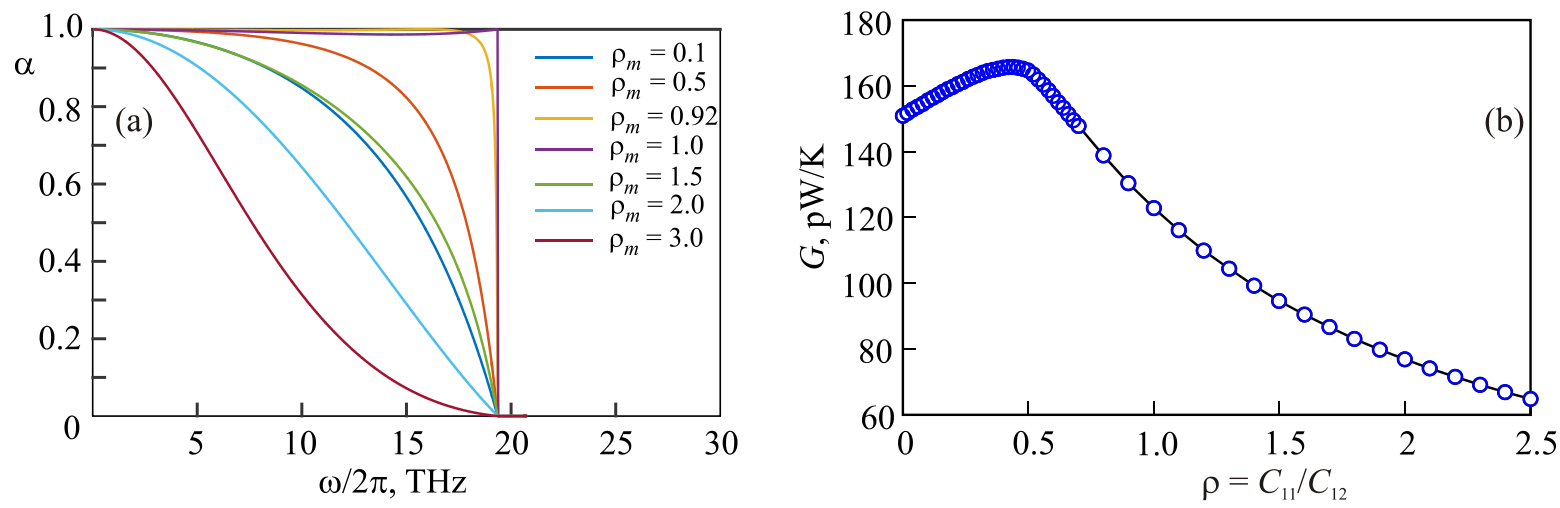

Fig. 3. (Color online) (a) Phonon transmission $\alpha$ of the quasi-1D model with an impurity atom in Fig. 1 vs frequency for different impurity mass ratio $\rho_{m}$. The force constant ratio $\rho=0.058$. The isotope of mass $m_{2}$ is coupled to the host atoms of mass $m_{1}$ and $\rho_{m}=m_{2} / m_{1}$ with $m_{1}=m_{\mathrm{Si}}$. (b) Thermal conductance $G\left(\rho_{m}\right)$ vs the impurity mass ratio $\rho_{m}$ at $T=300 \mathrm{~K}$. 

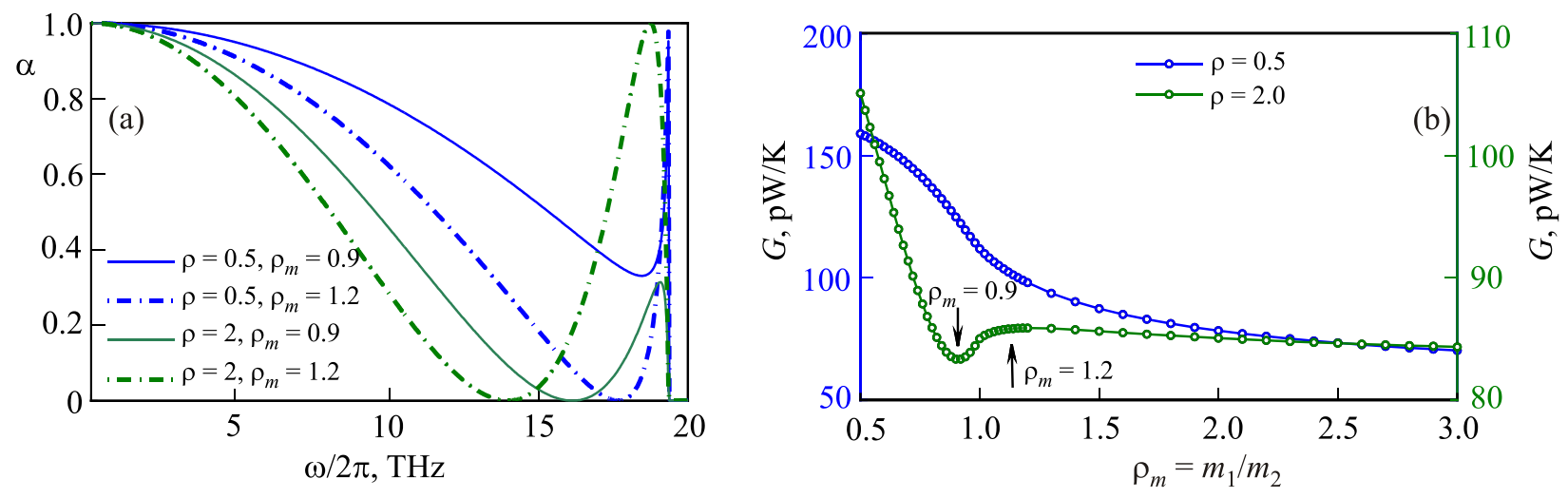

Fig. 4. (Color online) (a) Phonon transmission $\alpha$ of the quasi-1D model with a heavy impurity atom in Fig. 1(b) vs frequency for impurity mass ratio $\rho_{m}=0.9,1.2$ and for second NN bond strengths of $\rho=0.5,2$. (b) Thermal conductance $G$ vs the impurity mass ratio $\rho_{m}$ at $T=300 \mathrm{~K}$ for for second $\mathrm{NN}$ bond strength of $\rho=0.5$ and 2 .

The corresponding phonon transmission spectra are shown in Fig. 4(a). The transition of thermal conductance $G\left(\rho_{\mathrm{m}}\right)$ from its local minimum to the maximum is due to the increased transmission of high frequency phonons, similar to what was found in Fig. 1(a).

\section{Conclusion}

In conclusion, we investigate the role of second nearest neighbor interatomic forces on the thermal conductance of a silicon-germanium-like 2D metasurface. Controlled by the ratio between the second and first nearest-neighbor harmonic force constants, the thermal conductance across a germanium atomic plane in the silicon lattice exhibits a trend switch induced by the destructive interference of the nearest-neighbor phonon path with a direct path bypassing the defect atoms. We show that the heavy isotope impurity bypassed by long range interatomic bonds is crucial for the realization of the local minimum in the thermal conductance. We highlight the effect of the second phonon path on the distinct behaviors of the dependence of the thermal conductance on the impurity mass ratio. All our conclusions are confirmed both by Green's Function calculations for the equivalent quasi-1D lattice models and by molecular dynamics simulations.

\section{Appendix A: Phonon wave-packet technique using molecular dynamics}

To probe the phonon transmission, MD with the phonon wave packet method was used to provide the per-phononmode energy transmission coefficient $\alpha(\omega, l)$. We excited a realistic 3D Gaussian wave packet centered at the frequency $\omega$ and wave vector $\mathbf{k}$ in the reciprocal space and at $\mathbf{r}_{0}$ in the real space, with the spatial width (coherence length) $l$ in the direction of $\mathbf{k}$. The generation of the WP was performed by assigning the displacement $\mathbf{u}_{i}$ for the atom $i$ as:
$\mathbf{u}_{i}=A \mathbf{e}_{i}(\mathbf{k}) \exp \left(\mathrm{i}\left[\mathbf{k} \cdot\left(\mathbf{r}_{i}-\mathbf{r}_{0}\right)-\omega t\right]\right) \exp \left(-\frac{\left[\mathbf{r}_{i}-\mathbf{r}_{0}-\mathbf{v}_{g} t\right]^{2}}{4 l^{2}}\right)$,

where $A$ is the wave packet amplitude, $\mathbf{e}_{i}(\mathbf{k})$ is the phonon polarization vector, $\omega$ is the eigenfrequency for the wave vector $\mathbf{k}$ within a single branch of the phonon dispersion curve, $\mathbf{v}_{g}$ is the phonon group velocity along the wave vector $\mathbf{k}$ at the wave packet center frequency $\omega$. Wave amplitude $A$ of the generated phonon wave packets was taken sufficiently small such that the anharmonic coupling to other lattice modes is kept weak. Hence the wave packets propagate in an effectively harmonic crystal without any perceptible spreading or scattering. The wave packet was set to propagate normally to the defect layer, where an elastic scattering results in transmitted and reflected waves. The wave packet energy transmission coefficient $\alpha(\omega, l)$ is defined as the ratio between the energy carried by the transmitted and initial wave packets, centered at the given phonon mode $(\omega, \mathbf{k})$ with the spatial extent $l$. The planewave limit is reproduced by the wave packets with the spatial width $l$ much larger than the wavelength $\lambda_{c}$ of the wave packet central frequency. All the MD simulations were performed with the LAMMPS code package.

\section{Appendix B: Harmonic force constants determination from First Principle calculations}

We calculate the harmonic interatomic force constants (IFCs) of silicon by using a real-space displacement method combined with First Principle calculations [31]. The First Principle calculations were carried out using the quantum chemistry DFT code Quantum ESPRESSO [32]. The calculation was conducted for a $2 \times 2 \times 2$ supercells of bulk silicon containing 64 atoms by the plane-wave basis method implemented in the Quantum Espresso package. We adopted both generalized gradient approximation 
(GGA) for the exchange-correlation functional to study pseudopotential-dependent phonon properties. The parameterizations of Perdew-Burke-Ernzerhof were selected for GGA. The cutoff energies for plane-wave expansion was set to 60 Ry $(\approx 816 \mathrm{eV})$ for GGA. A Monkhorst-Pack k-point grid of $4 \times 4 \times 4$ was used to achieve the desired accuracy. Constraints due to translational invariance and other symmetries were employed to identify the minimum number of independent IFCs that needed to be computed. We have set the range of harmonic IFCs to five nearest neighbor shells. This results in 17 independent harmonic IFCs. The IFCs were fitted with the ALAMODE package [33].

\section{Appendix C:Thermal conductance and phonon transmission in a 1D model using atomistic Green's function}

In the scattering theory, the system contains three coupled subsystems: two semi-infinite leads connected through the scattering region. The heat flux flowing in along the system axis writes

$$
J=\int_{B Z} \hbar \omega_{\mathbf{k}} v_{g, k_{z}}\left(n_{L}-n_{R}\right) t_{\mathbf{k}} \frac{d^{3} \mathbf{k}}{(2 \pi)^{3}}
$$

where $\hbar \omega_{\mathbf{k}}$ is the energy quantum of the phonon mode $\mathbf{k}$, $v_{g, k_{z}}$ is the phonon group velocity of the phonon mode $k_{z}$, $n_{L, R}$ is the phonon number on the left and right reservoir following the Bose-Einstein distribution $n=\left(\mathrm{e}^{\frac{\hbar \omega}{k_{B} T}}-1\right)^{-1}$, $t_{\mathbf{k}}$ is the normalized transmission probability of the phonon mode $\mathbf{k}$ and $t_{\mathbf{k}} \in[0,1]$. The integration goes through all the phonon modes in the irreducible Brillouin Zone (BZ). In the linear regime, the phonon population undergoes small perturbations and thus the thermal conductance writes

$$
G=J / \Delta T=\int_{B Z} \hbar \omega_{\mathbf{k}} v_{g, k_{z}} \frac{\partial n}{\partial T} t_{\mathbf{k}} \frac{d^{3} \mathbf{k}}{(2 \pi)^{3}} .
$$

We note that $d^{3} \mathbf{k}=d k_{x} d k_{y} d k_{z}$ and $v_{g, k_{z}} d k_{z}=$ $=\partial \omega / \partial k_{z} d k_{z}=d \omega$. The Eq. (C.2) reduces to

$G=J / \Delta T=\int_{B Z} \hbar \omega \frac{\partial}{\partial T}\left(\mathrm{e}^{\frac{\hbar \omega}{k^{T}}}-1\right)^{-1}\left[t_{\omega} d k_{x} d k_{y}\right] \frac{d \omega}{(2 \pi)^{3}}$.

Hence we identify the spectral phonon transmission function $\Xi(\omega)=t_{\omega} g(\omega)$ where $g(\omega)=d k_{x} d k_{y}$ is the projected phonon density of states in the nonperiodic directions of the system.
We probe the spectral phonon transmission function $\Xi(\omega)$ by atomistic Green's Function (AGF) and the thermal conductance can be obtained by following the Landauer formula:

$$
G=\int_{0}^{\omega} \Xi(\omega) \frac{\partial}{\partial T}\left(\mathrm{e}^{\frac{\hbar \omega}{k_{B} T}}-1\right)^{-1} \hbar \omega \frac{d \omega}{2 \pi}
$$

where $\omega$ and $\omega_{\max }$ are the energy and the Debye frequencies. $T$ refers to the mean temperature of the system, $k_{B}$ and $\hbar$ represent the Boltzmann and the reduced Planck's constants, respectively. The transmission $\Xi(\omega)$ is obtained from a nonequilibrium Green's function approach as $\operatorname{Tr}\left[\Gamma_{L} G_{S} \Gamma_{R} G_{S}^{+}\right]$. The advanced and retarded Green functions $G_{S}^{+}$and $G_{S}$ can be deduced from

$$
G_{S}=\left[(\omega+i \Delta)^{2} I-K_{s}-\Sigma_{L}-\Sigma_{R}\right]^{-1}
$$

where $\Delta$ is an infinitesimal imaginary part that maintains the causality of the Green's function and $\Sigma_{L}=K_{a b} g_{L} K_{a b}^{+}$, $\Sigma_{R}=K_{a b} g_{R} K_{a b}^{+}$are the self-energies of the left and right leads, the "+" exponent indicating the Hermitian conjugation. Finally, $g_{L}$ and $g_{R}$ refer to the surface Green's functions of the left and the right leads, while $K_{s}$ and $K_{a b}$ are the force constant matrices derived from the potential, for the scattering region and between neighboring atoms in the lead, respectively. The expression of the transmission also includes $\Gamma_{L}=i\left(\Sigma_{L}-\Sigma_{L}^{+}\right)$and $\Gamma_{R}=i\left(\Sigma_{R}-\Sigma_{R}^{+}\right)$.

In the quasi-1D tight-binding model of the Fig. 1(b) the self-coupling matrix of the scattering region $K_{S}$ writes,

$$
K_{S}=\left(\begin{array}{ccc}
\frac{C_{0}+C_{11}+C_{12}}{m_{1}} & -\frac{C_{12}}{\sqrt{m_{1} m_{2}}} & -\frac{C_{11}}{m_{1}} \\
-\frac{C_{12}}{\sqrt{m_{2} m_{1}}} & \frac{2 C_{12}}{m_{2}} & -\frac{C_{12}}{\sqrt{m_{2} m_{1}}} \\
-\frac{C_{11}}{m_{1}} & -\frac{C_{12}}{\sqrt{m_{1} m_{2}}} & \frac{C_{0}+C_{11}+C_{12}}{m_{1}}
\end{array}\right) .
$$

The matrix $K_{a b}^{L}$ coupling the scattering region to the left lead writes,

$$
K_{a b}^{L}=\left(-\frac{C_{0}}{m_{0}} \quad 0 \quad 0\right) .
$$

The matrix $K_{a b}^{R}$ coupling the scattering region to the right lead writes,

$$
K_{a b}^{R}=\left(\begin{array}{lll}
0 & 0 & -\frac{C_{0}}{m_{0}}
\end{array}\right) .
$$

In the calculations, the $\mathrm{NN}$ bonds in the host lead $C_{0}$ equals to that in the scattering region $C_{12}$. 
1. P.L. Kapitza, J. Phys. 4, 181 (1941).

2. I.M. Khalatnikov, An Introduction to the Theory of Superfludity, Addisson-Wesley (1989).

3. E.T. Swartz and R.O. Pohl, Rev. Mod. Phys. 61, 605 (1989).

4. T. Markussen, J. Chem. Phys. 139, 244101 (2013).

5. Yu.A. Kosevich, A. Feher, and E.S. Syrkin, Fiz. Nizk. Temp. 34, 725 (2008) [Low Temp. Phys. 34, 575 (2008)].

6. Yu. A. Kosevich, Phys. Usp. 51, 848 (2008).

7. H. Han, L.G. Potyomina, A.N. Darinskii, S. Volz, and Yu.A. Kosevich, Phys. Rev. B 89, 180301(R) (2014).

8. Yu.A. Kosevich, H. Han, L.G. Potyomina, A.N. Darinskii, and S. Volz, in: Quodons in Mica, Springer International Publishing (2015), p. 247.

9. H. Han, B. Li, S. Volz, and Yu.A. Kosevich, Phys. Rev. Lett. 114, 145501 (2015).

10. A. Fellay, F. Gagel, K. Maschke, A. Virlouvet, A. Khater, Phys. Rev. B 55, 1707 (1997).

11. Yu.A. Kosevich, Prog. Surf. Sci. 55, 157 (1997).

12. Zhaojie Wang, Joseph E. Alaniz, Wanyoung Jang, Javier E. Garay, and Chris Dames, Nano Lett. 11, 2206 (2011).

13. Kanishka Biswas, Jiaqing He, Ivan D. Blum, Chun-I Wu, Timothy P. Hogan, David N. Seidman, Vinayak P. Dravid, and Mercouri G. Kanatzidis, Nature 489, 7416 (2012).

14. J. Ma, O. Delaire, A.F. May, C.E. Carlton, M.A. McGuire, L.H. VanBebber, D.L. Abernathy, G. Ehlers, Tao Hong, A. Huq, Wei Tian, V.M. Keppens, Y. Shao-Horn, and B.C. Sales, Nature Nanotechnol. 8, 445 (2013).

15. N. Mingo, D. Hauser, N.P. Kobayashi, M. Plissonnier, and A. Shakouri, Nano Lett. 2, 711 (2009).

16. G. Pernot, M. Stoffel, I. Savic, F. Pezzoli, P. Chen, G. Savelli, A. Jacquot, J. Schumann, U. Denker, I. Mönch, Ch. Deneke, O.G. Schmidt, J.M. Rampnoux, S. Wang, M. Plissonnier, A. Rastelli, S. Dilhaire, and N. Mingo, Nat. Mater. 6, 491 (2009).

17. P. Chen, N.A. Katcho, J.P. Feser, W. Li, M. Glaser, O.G. Schmidt, D.G. Cahill, N. Mingo, and A. Rastelli, Phys. Rev. Lett. 111, 115901 (2013).
18. S. Plimpton, J. Comput. Phys. 117, 1 (1995).

19. F.H. Stillinger and T.A. Weber, Phys. Rev. B 31, 5262 (1985).

20. N. Mingo and L. Yang, Phys. Rev. B 68, 245406 (2003).

21. X.W. Wang, H. Lee, Y.C. Lan, G.H. Zhu, G. Joshi, D.Z. Wang, J. Yang, A.J. Muto, M.Y. Tang, J. Klatsky, S. Song, M.S. Dresselhaus, G. Chen, and Z.F. Ren, Appl. Phys. Lett. 93, 193121 (2008).

22. B. Weinstein and G. Piermarini, Phys. Rev. B 12, 1172 (1975).

23. Thermal Conductivity: Theory, Properties, and Applications, Terry M. Tritt (ed.) Kluwer Academic, New York (2004).

24. R.J. Bell and P. Dean, Discuss. Faraday Soc. 50, 55 (1970).

25. A. Debernardi, S. Baroni, and E. Molinari, Phys. Rev. Lett. 75, 1819 (1995).

26. D.C. Wallace, Thermodynamics of Crystals, Dover Books on Physics, Dover, New York (1998).

27. L. Lindsay, D.A. Broido, and T.L. Reinecke, Phys. Rev. Lett. 109, 095901 (2012).

28. Takuma Shiga, Junichiro Shiomi, Jie Ma, Olivier Delaire, Tomasz Radzynski, Andrzej Lusakowski, Keivan Esfarjani, and Gang Chen, Phys. Rev. B 85, 155203 (2012).

29. B.L. Davis and Mahmoud I. Hussein, Phys. Rev. Lett. 112, 055505 (2014).

30. C.W. Li, J. Ma, H.B. Cao, A.F. May, D.L. Abernathy, G. Ehlers, C. Hoffmann, X. Wang, T. Hong, A. Huq, O. Gourdon, and O. Delaire, Phys. Rev. B 90, 214303 (2014).

31. K. Esfarjani, G. Chen, and H.T. Stokes, Phys. Rev. B 84, 085204 (2011).

32. P. Giannozzi, S. Baroni, N. Bonini, M. Calandra, R. Car, C. Cavazzoni, D. Ceresoli, G.L. Chiarotti, M. Cococcioni, I. Dabo, and A. Dal Corso, J. Phys.: Condens. Matter 21, 395502 (2009).

33. T. Tadano, Y. Gohda, and S. Tsuneyuki, Phys. Rev. Lett. 114, 095501 (2015). 